\title{
Spin Physics at HERMES
}

\author{
M. Contalbrigo \\ (on behalf of the HERMES collaboration) \\ INFN and University of Ferrara, Via Saragat 1 - Blocco C, 44100 Ferrara - Italia
}

\begin{abstract}
The HERMES experiment at DESY is a second generation experiment to study the spin structure of the nucleon by measuring not only inclusive but also semi-inclusive and exclusive processes in deep-inelastic lepton scattering where both beam and target can be polarised. An overview of finalized measurements and an outlook to upcoming new results is given for data taken with hydrogen and deuterium targets with longitudinal and transverse nucleon polarisation.
\end{abstract}

Keywords: nucleon structure, spin physics, parton distributions, single-spin asymmetries, DVCS PACS: 14.20.Dh, 13.60.Fz, 13.60.Hb,13.60.Le, 13.60.-r, 13.8.+e

\section{INTRODUCTION}

The nucleon spin can be decomposed into the contribution of nucleon constituents according to

$$
\left\langle s_{z}^{N}\right\rangle=\frac{1}{2}=\frac{1}{2} \Delta \Sigma+\Delta g+L_{q}+L_{g},
$$

where the four terms are the contributions from quark $\Delta \Sigma$ and gluon $\Delta g$ spins and the total angular momenta of the quarks $L_{q}$ and gluons $L_{g}$, respectively. All contributions are scale and scheme dependent. From early measurements it was realized that simple leading order considerations were too naive. Next-to-leading order treatments provide a picture more consistent with our present understanding of QCD but are not conclusive yet based on the available data. Further progress needs more accurate measurements of the individual contributions of Equation 1 to the nucleon spin.

The Hermes deep-inelastic scattering (DIS) experiment [1] has been taking data at the HERA accelerator in Hamburg from 1995 to 2007, aiming to get information on all the parton contributions to the nucleon spin. From inclusive DIS measurements it is possible to obtain the polarised structure function $g_{1}$ related to $\Delta \Sigma$; from semiinclusive DIS (SIDIS) measurements with observation of the produced hadron, the helicity distribution of individual quark flavors $\Delta q$ and gluons $\Delta g$ in the nucleon can be extracted; the possible role of quark orbital angular momentum is addressed by studying exclusive reactions; finally, by measuring DIS off a transversely polarised target, it is possible to investigate fundamental phenomena related to transverse spin effects in the nucleon.

At HERMES, longitudinally polarised electron or positron beams of $27.5 \mathrm{GeV}$ scatter from longitudinally or transversely polarised targets internal to the beam pipe. The positrons (electrons) circulating in the ring become transversely polarised by the emission of synchrotron radiation [2]. Longitudinal beam polarisation is achieved at HERMES by the use of a pair of spin rotators before and after the experiment. A feature of HERMES

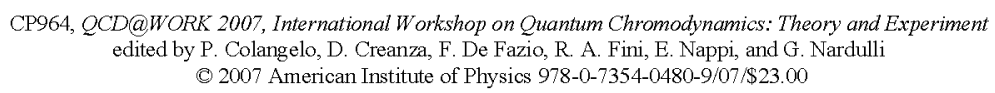


unique among polarised DIS experiments is its pure atomic-gas target that is not diluted by non-polarisable material [3]. A momentum resolution better than $2 \%$ for electrons is provided by the forward spectrometer. Electron/hadron separation is accomplished using a likelihood method based on signals of three subsystems: a lead-glass calorimeter, a transition-radiation detector, and a preshower hodoscope. The Ring Imaging Cerenkov detector provides full separation between charged pions, kaons and protons over essentially the entire momentum range of the experiment $(2$ to $15 \mathrm{GeV} / \mathrm{c})$.

\section{INCLUSIVE ASYMMETRIES}

The final results for the polarised structure function $g_{1}\left(x, Q^{2}\right)$ are obtained from a refined analysis of data taken with longitudinally polarised hydrogen and deuterium targets in the kinematic range $Q^{2}>0.1 \mathrm{GeV}^{2}$ and $W>1.8 \mathrm{GeV}$ [4], see Figure 1 (left panel). The variable $Q^{2}$ is the negative squared four-momentum transferred by the virtual photon, $x$ is the fraction of the nucleon's light-cone momentum carried by the struck quark, $W$ is the invariant mass of the photon-nucleon system. The statistical precision of the proton data is comparable to that of the hitherto most precise data from SLAC and CERN in the same $x$ range, while the deuteron data provide the most precise determination of $g_{1}^{d}$ compared to previous measurement in the same kinematic domain. Important information about the spin structure of the nucleon can be obtained from the
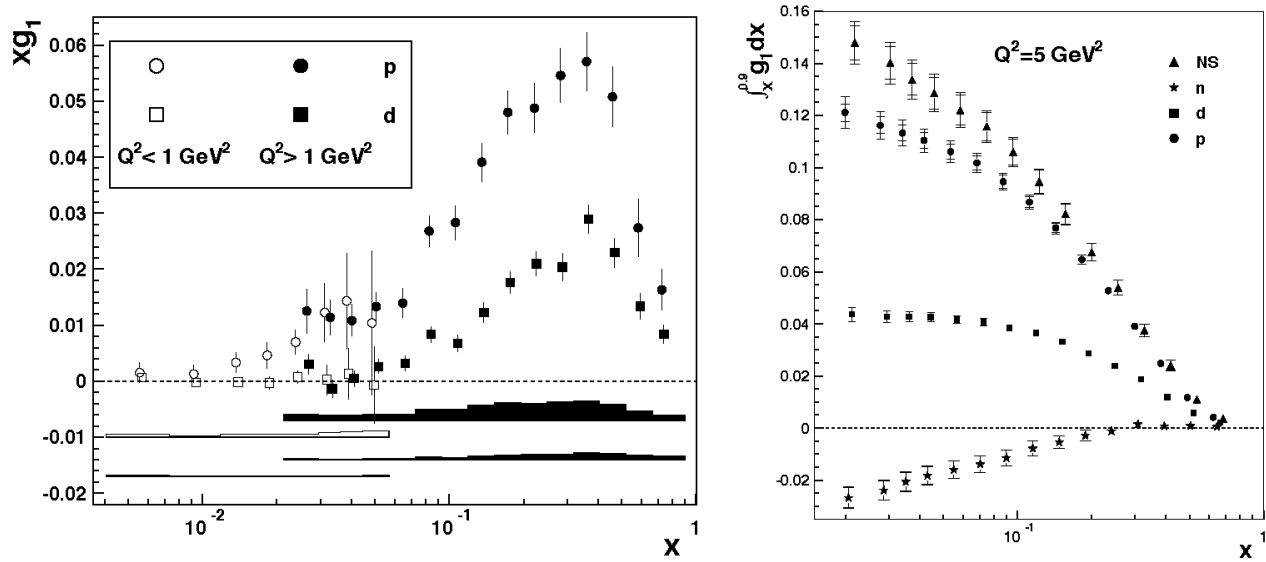

FIGURE 1. (Left) HERMES results for $x g_{1}$ vs $x$ for the proton and the deuteron. The data points are statistically correlated since the measured asymmetries have been corrected for detector smearing and QED radiative effects. The systematic error (lower bands) is dominated by the target and beam polarisation uncertainty. (Right) Integrals of $g_{1}^{p, d, n}$ and $g_{1}^{N S}=g_{1}^{p}-g_{1}^{n}$ evaluated at $Q_{0}^{2}=5 \mathrm{GeV}^{2}$ over the range $0.021<x<0.9$, corresponding to the event selection $Q^{2}>1$ $\mathrm{GeV}^{2}$, as a function of the low- $x$ limit of integration.

first moment of $g_{1}$, in particular the singlet distribution $\Delta \Sigma$ can be inferred under the assumption of SU(3) symmetry. For $x<0.04, g_{1}^{d}$ becomes compatible with zero and its measured integral shows saturation, see Figure 1 (right panel). This observation is supported by data at larger energy scale [5]. Based on the assumed saturation of the 
integral of $g_{1}^{d}$, the flavor-singlet axial charge has been determined in the $\overline{\mathrm{MS}}$ scheme at $Q_{0}^{2}=5 \mathrm{GeV}^{2}$, by using only the $g_{1}^{d}$ integral and the axial charge $a_{8}$ as inputs: $\mathrm{a}_{0}=0.330 \pm 0.011$ (theo) \pm 0.025 (exp.) \pm 0.028 (evol.). This result is in nice agreement with another extraction recently done [6], and can be interpreted as the contribution $\Delta \Sigma$ of quark spins to the spin of the nucleon in $\overline{\mathrm{MS}}$ factorisation scheme. The data therefore suggest that the quark helicities contribute a substantial fraction to the nucleon helicity, but there is still need for a major contribution from gluons and/or orbital angular momenta of more than half of the sum of Equation 1.

\section{QUARK AND GLUON HELICITY DISTRIBUTIONS}

In principle, indirect access to the polarised gluon and sea-quark distributions is provided by a NLO QCD analysis of the scaling violations of the polarised structure function $g_{1}$. In contrast to the unpolarised case, the limited data statistics and the restricted $Q^{2}$ range covered by the experiments so far do not constrain significantly neither the gluon nor the sea-quark polarised distributions. Semi-inclusive deep-inelastic scattering is a powerful tool to determine the separate contributions $\Delta q\left(x, Q^{2}\right)$ of the quarks and antiquarks of flavor $q$ to the total spin of the nucleon. The measured semi-inclusive photon-nucleon asymmetry $A_{1}^{h}$ for the hadron of type $h$ is related to the quark and antiquark polarisations $\Delta q\left(x, Q^{2}\right)$ through the so-called purity matrix $P_{q}^{h}$, representing the probability that the quark $q$ was struck in the DIS event with detected hadron $h$. The purities are obtained from Monte Carlo simulations based on the Lund string fragmentation model tuned to Hermes kinematics. For the first time, an analysis of the inclusive spin asymmetries together with the semi-inclusive spin asymmetries for $\pi^{+}, \pi^{-}, K^{+}$and $K^{-}$has been carried out for longitudinally polarised targets of hydrogen and deuterium [7]. No assumptions have been made on the symmetry of the sea flavors, except that the poorly constrained $\Delta \bar{s}$ is assumed to be zero. The results of the decomposition are presented in Figure 2 (left panel). As expected, the results show that $\Delta u(x)$ is positive and $\Delta d(x)$ is negative, and that both reach their maximum of magnitude in the valence quark region. The polarised sea quark distributions are all consistent with zero. The net contribution of quark spins to the nucleon spin, when explained under the assumption of SU(3) flavor symmetry, favors a significant negative value for the strange quark polarisation. Such a negative value would explain the violation of the Ellis-Jaffe sum rule in inclusive DIS. The polarisation of the strange quarks in the proton is therefore of particular interest. In the measured range, the polarised sea quark distributions extracted from flavor decomposition show no disagreement with the results of LO QCD analyses of inclusive data within the experimental uncertainties [8,9]. Any significant negative contribution of the strange polarisation should therefore concentrate at lower value of $x$. Since the total strange quark helicity density $\Delta S(x) \equiv \Delta s(x)+\Delta \bar{s}(x)$ carries no isospin, it can be extracted from a measurement on deuterium alone, which is isoscalar, by assuming only charge-conjugation invariance in fragmentation [10]. The preliminary result of a refined analysis indicates a strange quark polarisation compatible with zero, in agreement with the results based on the combined analysis of the proton and deuteron data described above and already published [7]. 

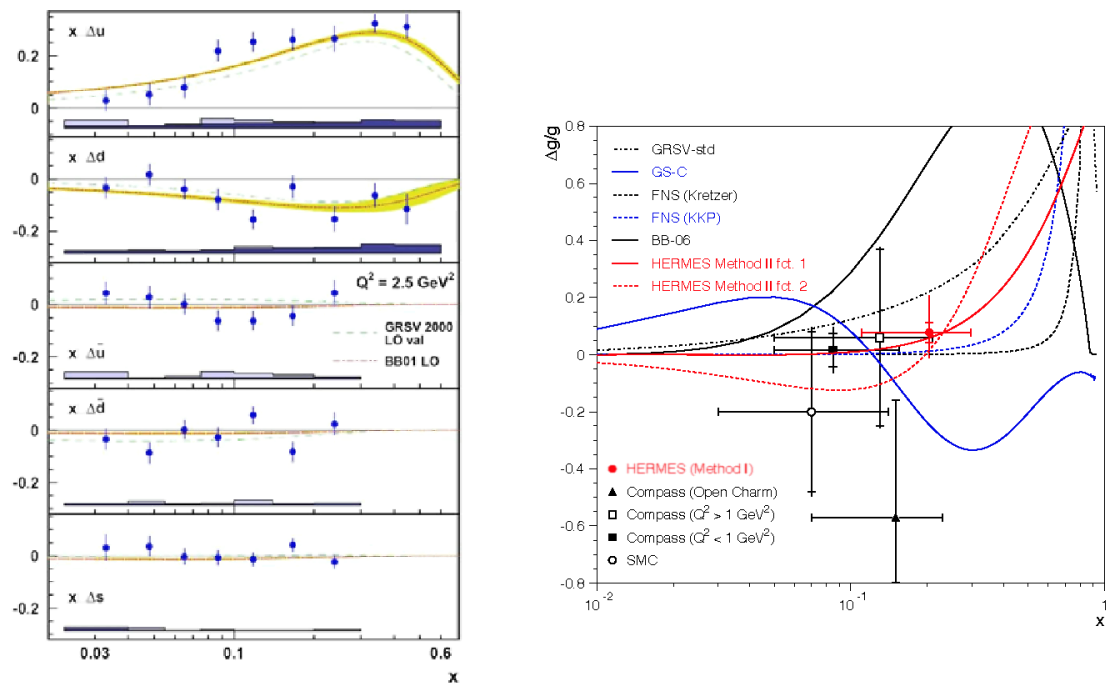

FIGURE 2. (Left) The $x$-weighted polarised quark densities. The errors are presented as in Figure 1 . The plots show a five parameter fit to the data assuming $\Delta \bar{s}=0$. The data have been evolved to a common value of $Q_{0}^{2}=2.5 \mathrm{GeV}^{2}$. The curves show LO QCD analyses fits to the inclusive world polarised data set $[8,9]$. (Right) HERMES results for $\Lambda g / g(x)$ together with world DIS data compilation $[11,12]$. The experimental systematic error from beam and target polarisations is small compared to the model uncertainty estimated by varying the Pythia model parameters and assumptions. The curves represent two fit functions from Method II (see text) and results from several NLO-QCD fits $[8,9,13,14]$.

A direct LO, model dependent extraction of $\Delta g / g$ has been performed by studying charged hadron production at large transverse momenta $p_{T}$. The high statistic data sample of antitagged (vetoed by the beam particle) inclusive charged hadrons was used. To relate the measured longitudinal double-spin asymmetry to the gluon polarisation $\Delta g / g$, information on the relative contributions of the various subprocesses to the inclusive hadron production cross-section, their asymmetries and variation with $p_{T}$ has been estimated from detailed Monte Carlo simulation using Pythia 6.2 and parametrization of the spin dependent parton distributions of the nucleon and the photon. This information is used to extract the signal asymmetry which still contains a convolution of $\Delta g / g$ with the hard subprocess cross-section. Two methods have been applied to extract the average $\langle\Delta g / g\rangle\left(p_{T}\right)$ from the signal asymmetry using different assumptions on the shape of $\Delta g(x) / g(x)$. Method I assumes that $\Delta g(x) / g(x)$ is constant in the measured $x$ range while Method II employs a functional form for $\Delta g(x) / g(x)$. From Method II and by integrating over $1.05<p_{T}<2.5 \mathrm{GeV}$, a value of $\Delta \mathrm{g} / \mathrm{g}=0.071 \pm 0.034(\mathrm{stat}) \pm 0.010(\mathrm{sys}-\mathrm{exp})_{-0.105}^{+0.127}(\mathrm{sys}-$ models $)$ has been obtained at $\langle x\rangle=0.22$ and $\left\langle\mu^{2}\right\rangle=1.35 \mathrm{GeV}^{2}$. This result is shown in Figure 2 (right panel) together with previous determinations of $\Delta g / g[11,12]$ and is compared to several different parameterizations obtained from NLO-QCD fits $[8,9,13,14]$. The data favor a very small 
$\Delta g$, or the presence of a $\Delta g(x)$ node at $x$ around 0.1 .

\section{TRANSVERSE SPIN EFFECTS}

A complete description of the quark structure of the nucleon in leading twist requires in addition to the unpolarised distribution $q\left(x, Q^{2}\right)$ and the helicity distribution $\Delta q\left(x, Q^{2}\right)$ a third quark distribution, named transversity $\delta q\left(x, Q^{2}\right)$. The latter corresponds to a number density reflecting the difference in probabilities to find quarks in a transversely polarised nucleon with their spin aligned or anti-aligned to the spin of the nucleon. In a basis of helicity eigenstates, it is related to a forward scattering amplitude involving helicity flip of both quark and target nucleon. Since hard interaction preserve chirality, transversity cannot be measured in inclusive processes due to its chiral-odd nature, but only in a process in which it is combined with another chiral-odd object. The most direct approach is to measure transversity from double transverse-spin asymmetries in polarised Drell-Yan processes [15]. In DIS off a transversely polarised nucleon target, transversity enters the cross-section combined with novel chiral-odd fragmentation functions (FFs). The Collins fragmentation function $H_{1}^{-}(z)$ describes a spin momentum correlation where the transverse polarisation of the struck quark influences the transverse momentum component of the produced hadron orthogonal to the virtual photon, thereby leading to an azimuthal dependence of the cross-section. Azimuthal spin asymmetries can be also generated by the T-odd Sivers distribution function $f_{1}^{\perp}\left(x, k_{T}\right)$ that appears in the cross-section together with the unpolarised FF $D(z)$. The Sivers distribution describes a correlation between the transverse polarisation of the target nucleon and the intrinsic transverse momentum $k_{T}$ of the struck quark. Its existence requires non-zero orbital angular momentum of quarks. The Collins and Sivers functions are example of transverse momentum dependent distributions (TMDs) which are under intense investigation since they provide new insight into the nucleon intrinsic dynamics. In DIS off a transversely polarised nucleon target, they can be separated thanks to their different azimuthal dependences with respect of the axis of polarisation. While the Sivers mechanism gives rise to a $\sin \left(\phi-\phi_{S}\right)$ behavior, the Collins mechanism exhibits a $\sin \left(\phi+\phi_{S}\right)$ behavior, where $\phi$ is the azimuthal angle between the hadron production plane and the lepton scattering plane and $\phi_{S}$ the angle between the lepton scattering plane and the transverse spin component of the target nucleon. The first measurement of non-zero Collins and Sivers moments for charge pions as a function of $x, z$ and transverse hadron momentum $P_{h \perp}$ has been published by HERMES [16] based on a subsample of the collected data. The preliminary result on the full data-set is presented in Figure 3. The Collins amplitude is positive for $\pi^{+}$and negative for $\pi^{-}$. The large absolute magnitude of the $\pi^{-}$asymmetry is surprising, suggesting a substantial disfavored Collins function with a sign opposite to that of the favored function. This observation is consistent with the measurements at BELLE [17] and COMPASS [18], provided that transversity for $u$ and $d$ quarks has opposite sign resembling the helicities densities [19]. The Sivers amplitude is significantly positive for $\pi^{+}$, providing an indication of non-zero orbital angular momentum of quarks in the nucleon and the first evidence for a T-odd distribution function, while it is consistent with zero for $\pi^{-}$. The naively T-odd Sivers function, as extracted in DIS reactions, can in future be compared to its appearance in polarised 

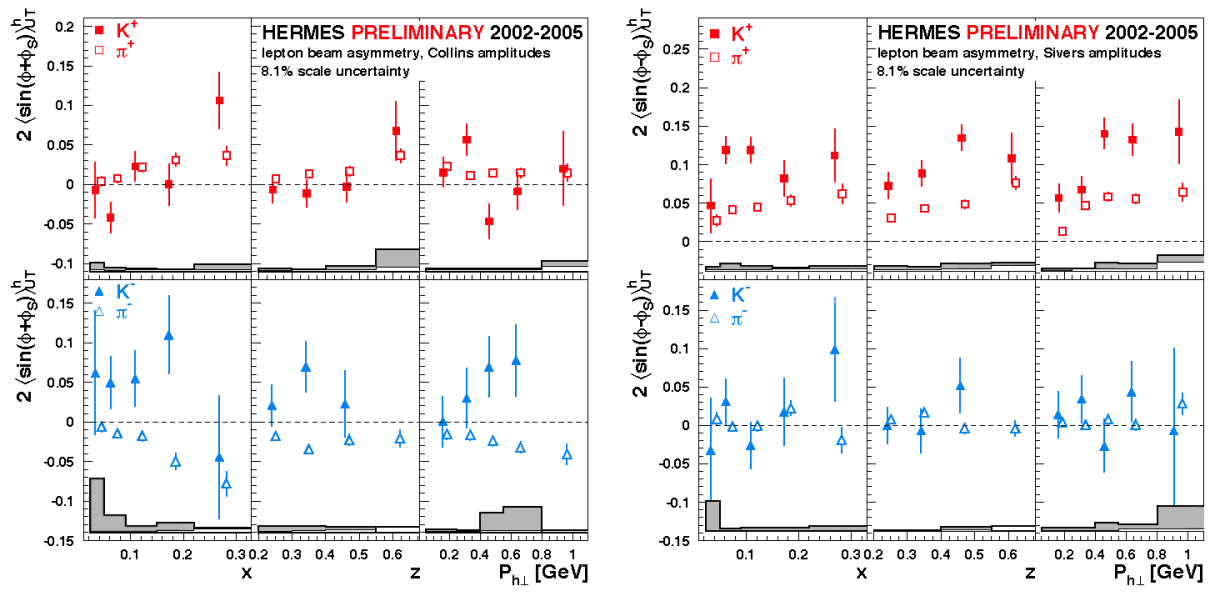

FIGURE 3. Collins (left) and Sivers (right) moments for charged pions and kaons as a function of $x, z$ and transverse hadron momentum $P_{h \perp}$. Error bars represent the statistical uncertainties, while the lower band represents the maximum systematic uncertainty due to acceptance and detector smearing as well as possible contributions from $\cos \phi$ moments of the unpolarised cross-section. Effects in acceptance, instrumental smearing and QED radiative were all found to be negligible in Monte Carlo simulations. The $6.6 \%$ scale uncertainty is due to the target polarisation.

Drell-Yan processes, where it is predicted to have opposite sign due to its fundamental time reversal invariance in QCD $[20,15]$. For the first time, these moments have been extracted also for the charged kaons, using a maximum likelihood based fit: the results are shown in Figure 3. While there is no reason to expect a similar amplitude for $\mathrm{K}^{-}$ and $\pi^{-}$, being a $K^{-}$a fully sea object, the $u$-quark dominance in DIS would suggest a similar amplitude for $K^{+}$and $\pi^{+}$. On the other hand, the amplitude of Sivers moment for $K^{+}$is roughly twice as big as that for $\pi^{+}$in the region $x \approx 0.1$. This suggests that the sea quarks may provide an important contribution to the Sivers function and thus may carry significant orbital angular momentum in the nucleon. Indeed, a recent parameterization of fragmentation functions, based on world data on DIS and hadron reaction experiments, in addition to the usual $e^{+} e^{-}$colliders data, favors an enhanced role of the $\bar{s}$ fragmentation into $K^{+}[21]$.

\section{EXCLUSIVE PROCESSES AND QUARK ORBITAL MOMENTUM}

Only recently the concept of Generalized Parton Distributions (GPDs) has been developed which allows to study the dynamics of quarks and gluons in a very general framework [22]. GPDs combine the features of form factors, parton densities and distribution amplitudes and allow to depict a 3-dimensional picture of the nucleon [23]. These parton correlation functions can give experimental access to the total angular momentum carried by the quarks $J_{q}$ in the nucleon [24]. Thus, with prior knowledge of $\Delta \Sigma$, the orbital 


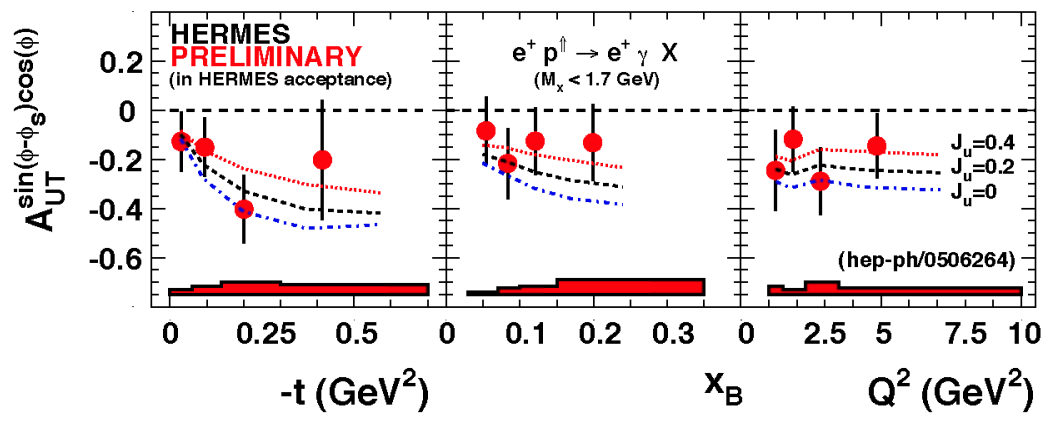

FIGURE 4. DVCS transverse target spin asymmetry amplitudes $A_{U T}^{\sin \left(\phi-\phi_{S}\right) \cos \phi}$ and $A_{U T}^{\cos (\phi-\phi S) \sin \phi}$, as measured by HERMEs. The curves shows the expectations for different values of the total angular momentum $J_{u}\left(J_{u}=0.0,0.2,0.4\right)$ and $J_{d}=0$ provided by a GPD model $[27,28]$.

angular momentum of the quarks $L_{q}$ may become accessible.

The cleanest way to access GPDs is Deeply Virtual Compton Scattering (DVCS), the production of a real photon in hard exclusive electron scattering. DVCS amplitudes can be determined through a measurement of the interference between the DVCS and Bethe-Heitler processes, in which the photon is radiated from a parton and from the lepton, respectively. Measuring the azimuthal dependence of the cross-section asymmetry with respect to the charge (spin) of the lepton beam provides information about the real (imaginary) part of the DVCS amplitude. HERMES has already measured azimuthal asymmetries with respect to the beam helicity [25] and to the beam charge [26], showing high sensitivity to different GPD parameterizations. DVCS transverse target-spin asymmetries (TTSA) give access to a combination of $H_{q}$ with unsuppressed contribution of $E_{q}$, the two GPDs which are related to the quark total angular momentum $J_{q}$ by the Ji sum rule [24]. Preliminary results on the amplitudes of DVCS TTSA obtained on about half of the accumulated HERMES statistics are shown in Figure 4. The data are compared with a model predictions parameterized as function of the total angular momentum $J_{u}$ [27]. Based on $u$-quark dominance in DIS reactions, the $d$-quark total angular momentum $J_{d}$ has been set to zero. Since the model calculations are largely insensitive to all model parameters but $J_{u}$ and $J_{d}$, it is possible to constrain $J_{u}$ and $J_{d}$, even though other model parameters are largely unknown [28].

At present, the recoiling target nucleon is not detected in the HERMES apparatus. Therefore the exclusivity of the process is ensured by the missing mass technique. At the beginning of 2006, the HERMES spectrometer was upgraded by a recoil detector to measure the recoil proton and thus identify the complete final state of the DVCS process. With improved kinematic resolution and background suppression, the model parameters for the GPD $H_{q}$ can be well constrained by the envisaged HERMES DVCS measurements until 2007 and, in conjunction with the transverse target spin data, provide insight on the unknown GPD $E_{q}$ and the total angular momentum $J_{q}$. 


\section{CONCLUSIONS}

In spite of many years of experimental effort, a detailed decomposition of the spin of the nucleon remains elusive. At HERMES, inclusive and semi-inclusive measurements with polarised beam and targets have provided novel information on the flavor decomposition of the quark and gluon helicity distributions and new insights in their contributions to the nucleon spin. Transverse spin effects have been investigated leading to the first observation of non-zero asymmetries due to Collins mechanism, related to transversity, and Sivers mechanism, related to the orbital angular momentum of quarks. The DVCS measurements on unpolarised and transversely polarised hydrogen target lead to a first model dependent constraint for the total angular momentum of quarks in the nucleon. At the beginning of 2006, a major upgrade of the HERMES spectrometer was the insertion of a recoil detector design to substantially improve measurements of hard exclusive production of mesons and real photons with positron and electron beams.

\section{REFERENCES}

1. Hermes Collab. (K. Ackerstaff et al.), Nucl. Instrum. Methods A 417, 230 (1998).

2. A. Sokolov and L. Ternov, Sov. Phys. Doklady 8, 1203 (1964).

3. Hermes Collab. (A. Airapetian et al.), Nucl. Instrum. Methods A 540,68 (2005).

4. Hermes Collab. (A. Airapetian et al.), Phys. Rev. D 75, 012007 (2007).

5. Compass Collab. (E.S. Ageev et al.), Phys. Lett. B 647, 330 (2007).

6. Compass Collab. (V.Yu. Alexakhin et al.), Phys. Lett. B 647, 8 (2007).

7. Hermes Collab. (A. Airapetian et al.), Phys. Rev. Lett. 92, 012005 (2004).

8. M. Glück et al., Phys. Rev. D 63, 094005 (2001).

9. J. Blümlein and H. Böttcher Nulc. Phys. B 636, 225 (2002).

10. H. Jackson, AIP Conf. Proc. 842, 363 (2006). Hermes Collab. (A. Airapetian et al.), Phys. Rev. D 71, 012003 (2005).

11. Compass Collab. (E.S. Ageev et al.), Phys. Lett. B 633, 25 (2006).

12. SMC Collab. (B. Adeeva et al.), Phys. Rev. D 70, 012002 (2004).

13. T. Gehrmann, W. Stirling, Z. Phys. C 65, 461 (1995).

14. D. de Florian, G. Navarro, R. Sassot, Phys. Rev. D 71, 094018(2005).

15. PAX Collab. (V. Barone et al.), e-Print hep-ex/0505054.

16. Hermes Collab. (A. Airapetian et al.), Phys. Rev. Lett. 94, 012002 (2005).

17. BELLE Collab. (K. Abe et al.), Phys. Rev. Lett. 96, 232002 (2006).

18. Compass Collab. (E.S. Ageev et al.), Nulc. Phys. B 765, 31 (2007).

19. M. Anselmino et al., Phys. Rev. D 75, 054032 (2007).

20. J.C. Collins, Phys. Lett. B 536, 43 (2002).

21. D. de Florian, R. Sassot, M. Stratmann, Phys. Rev. D 75, 114010 (2007).

22. M. Dittes et al., Phys. Lett. B 209, 325 (1988); D. Mueller et al., Fortsch. Phys. 42101 (1994); A.V. Radyushkin, Phys. Lett. B 385333 (1996).

23. M. Burkardt, Phys. Rev. D. 62, 071503 (2000), Erratum-ibid. 66, 119903 (2002).

24. X. Ji, Phys. Rev. Lett. 78, 610 (1997); X. Ji, Phys. Rev. D. 55, 7114 (1997).

25. Hermes Collab. (A. Airapetian et al.), Phys. Rev. Lett. 87, 182001 (2001).

26. Hermes Collab. (A. Airapetian et al.), Phys. Rev. D 75, 011103 (2007).

27. K. Goeke et al. Prog. Part. Nucl. Phys. 47, 401 (2001).

28. F. Ellinghaus, W.-D. Nowak, A.V. Vinnikov and Z. Ye, Eur. Phys. J C 46, 729 (2006). 